\title{
Effects of Event Rate and Display Time on Sustained Attention in Hyperactive, Normal, and Control Children
}

\author{
Phyllis Chee, ${ }^{1}$ Gordon Logan, ${ }^{2}$ Russell Schachar, ${ }^{3,5}$ Peter Lindsay, ${ }^{4}$ and \\ Rod Wachsmuth ${ }^{3}$
}

Two experiments were conducted to determine whether hyperactive boys have a unique deficit in sustained attention. Groups with DSM-III diagnoses of attention deficit disorder $(A D D H)$, conduct disorder $(C D), A D D H+C D$, and learning disorder were compared with normal controls on the Continuous Performance Task. In Experiment 1, stimulus presentation rate (stimulus onset asynchrony, $S O A$ ) and display time were varied to manipulate attentional demand, and speed and accuracy of performance were measured. The ADDH group was uniquely affected, with less accurate performance at the fastest and slowest SOA. To distinguish the effects of time on task and $S O A$, the duration of each $S O A$ condition was held constant in Experiment 2. The poorer performance of the $A D D H$ group at the fastest SOA

Manuscript received in final form November 2, 1988.

This research was supported by the Ontario Mental Health and Ruth Schwartz Foundations through a graduate fellowship awarded to P. Chee and a research grant to R. Schachar, G. Logan, and R. Wachsmuth. Dr. Schachar was also supported by the Canadian Psychiatric Research Foundation. This article is based, in part, on a doctoral dissertation submitted by the first author to the Ontario Institute for Studies in Education. The authors thank Dr. John Lovering and the staff of the Departments of Psychiatry and Psychology, The Hospital for Sick Children, for their cooperation in the conduct of this study. This paper was prepared with the assistance of the Medical Publications Department, The Hospital for Sick Children, Toronto.

${ }^{1}$ Board of Education, City of Toronto, 155 College St., Toronto, Ontario, Canada M5T 1P6. ${ }^{2}$ Department of Psychology, 603 E. Daniel St., University of Illinois at Champaign, Champaign, Illinois 61820 .

${ }^{3}$ Department of Psychiatry, The Hospital for Sick Children, 555 University Ave., Toronto, Ontario, Canada M5G 1 X8.

${ }^{4}$ Department of Special Education, Ontario Institute for Studies in Education, 252 Bloor St. W., University of Toronto, Toronto, Ontario, Canada M55 IV6.

${ }^{5}$ Address all correspondence to Dr. R. Schachar, Department of Psychiatry, The Hospital for Sick Children, 555 University Avenue, Toronto, Ontario, Canada M5G 1 X8. 
was no longer evident. This finding indicates that the deficit of sustained attention in boys who have ADDH is associated with a greater susceptibility to refractory effects, which is influenced by practice.

The underlying disability of hyperactive children is thought to be a deficit in the ability to focus and sustain attention and to resist impulsive responding during academic and social tasks (Douglas, 1983). This hypothesis appears to be supported by considerable clinical and experimental evidence, and especially by the finding that, compared with normal controls, these children perform poorly on attention-demanding laboratory tasks (for a review see Douglas, 1983). However, doubt remains about the validity of the hypothesis because of methodological problems characterizing many of the studies.

One problem is that poor performance on a single condition of a task has been attributed to attention deficit. With such a design, it is impossible to distinguish poor performance due to inattentiveness from that due to other variables, such as lack of comprehension or motivation. It would be more appropriate to measure attention in terms of the effects of variables known to alter attention. The magnitude of these effects (e.g., the difference between an "easy" and a "difficult" attention condition) can be compared between groups to see whether the groups differ in their ability to attend. Groups that are deficient in attention should show larger (or in some cases smaller) differences than "normal" groups. An attention deficit would appear as an interaction between the independent variable and diagnostic group (e.g., hyperactive patients vs. normal controls).

Furthermore, in most research, performance on sustained attention tasks such as the Continuous Performance Task (CPT; Rosvold, Mirsky, Sarason, Bransome, \& Beck, 1956) has been measured in terms of accuracy but not speed, and performance on reaction time (RT) tasks in terms of speed but not accuracy. Speed and accuracy must be measured simultaneously; otherwise, differences among experimental groups will not be detected when groups of subjects trade speed for accuracy differently, as has been seen in some studies (e.g., Firestone \& Martin, 1979; Sergeant \& Scholten, 1985).

In addition, there are problems in the diagnosis of hyperactivity. Typically, diagnosis has been based on the results of various questionnaires, clinical interviews, or direct observations that are not clearly described or may be of questionable validity (Schachar, Sandberg, \& Rutter, 1986; Sergeant, 1981; Shaffer \& Greenhill, 1979). A related difficulty arises from the existence of two sets of criteria for diagnosing hyperactivity: DSM-III (American Psychiatric Association, 1980) and ICD-9 (World Health Organization, 1978). Since these diagnostic schemata differ with respect to the pervasiveness of hyperactive symptomatology necessary for the diagnosis, they define different groups of children as hyperactive (Taylor, 1986). These groups 
could differ in attentiveness as they do in other characteristics (Schachar, Rutter, \& Smith, 1981).

Finally, most studies do not control for the presence of additional diagnoses (e.g., learning and conduct disorders) among hyperactive children, although this is currently recommended (Lambert \& Sandoval, 1980; Prior \& Sanson, 1986; Werry, Reeves, \& Elkind, 1987). These syndromes, which have all been associated with inattentiveness, frequently occur in the same child (Shaffer, McNamara, \& Pincus, 1974; Swanson, 1983). Moreover, previous studies have not distinguished hyperactivity with conduct disorder from hyperactivity without conduct disorder, despite evidence that they differ in important ways (Campbell \& Werry, 1986). Therefore, to identify an attention deficit unique to hyperactivity, it is necessary to compare hyperactive children with children who have conduct or learning disorder and are not hyperactive, with children who have conduct disorder and are hyperactive, and with normal children.

Keeping these problems in mind, we designed an experiment to determine whether hyperactive children exhibit a deficit in sustained attention and, furthermore, whether this deficit is unique to hyperactive children. In this experiment, we specified criteria for diagnosing hyperactivity, compared the predictive validity of DSM-III and ICD-9 diagnostic criteria, and compared hyperactive children with normal children and also with nonhyperactive children who had learning or behavioral disorders. Demand for sustained attention was also manipulated and performance assessed in terms of both speed and accuracy.

\section{EXPERIMENT 1}

Two variables that affect attention when subjects are required to monitor a succession of stimuli for a specific target are length of stimulus exposure (display time, DT) and event rate (stimulus onset asynchrony, SOA). When displays are brief, any wavering in attention is likely to result in a failure to detect task-relevant stimuli. With a short DT, subjects whose attention wanders should make more errors (fewer hits and more false alarms); however, the subjects may respond by paying more attention to the task in order not to miss targets. Consequently, a short DT may produce more errors when attention is off task but faster RT when attention is on task and the target is detected correctly (McGrath, Harabedian, \& Bruckner, 1968).

SOA also affects the subjects' allocation of attention to the task. If the event rate is so fast (i.e., SOA is short) that subjects cannot finish attending and responding to one stimulus before the next appears, performance will suffer. RT will be slower than normal and accuracy will be lower (i.e., lower hit rate and higher false alarm rate). Performance should improve as the event 
rate slows (SOA increases) because subjects will have more time to complete processing before the next stimulus appears. The period over which performance is poor, called the psychological refractory period, occurs when SOA is 0 to about 500 msec (Davis, 1957; Kantowitz, 1974; Welford, 1952). As SOA increases beyond $500 \mathrm{msec}$, there may be time for attention to wander between trials, causing performance to deteriorate when attention is off task. This tendency may be exaggerated in subjects whose attention wanders more than normal.

Analysis of moment-by-moment effects of variation in SOA and DT brings us close to the underlying attentional processes: The predictions follow directly from most models of attention and the effects of a deficiency in sustained attention over several minutes represent compounded momentby-moment effects, which are the usual focus of investigation (e.g., Sykes, Douglas, Weiss, \& Minde, 1971).

Children with hyperactivity, conduct disorder, and learning disability were compared with normal children on the X version of the CPT. We employed a factorial design involving three DTs and three SOAs. Groups that are deficient in sustained attention (e.g., hyperactive children) should show greater deterioration in the more demanding conditions, relative to normal controls and pathological groups that are not deficient in sustained attention. The deficits would appear as interactions between diagnostic group and the attentional factors in the experiment (i.e., between group and DT; between group and SOA; and between group, SOA, and DT).

\section{Method}

\section{Subjects}

The subjects in this experiment were 51 boys who had been referred to a child psychiatry outpatient department for learning or psychiatric problems, and 36 normal boys from a local school. Only boys were included because of the predominance of males among hyperactive children (sex ratio 3:1; Schachar et al., 1981). Normal controls (NC) were obtained through a local school board. Teachers were asked to nominate boys who did not show behavioral or learning problems and were known not to have serious medical or family difficulties. The lack of problems was confirmed by information from a number of sources: (a) teacher ratings on the Rutter-B rating scale (Rutter, 1967), SNAP checklist (W. E. Pelham, personal communication, 1981), and Conners Abbreviated Teachers Questionnaire (Conners, 1973); (b) parental ratings on a behavior questionnaire made up of the Rutter A-2 scale (Rutter, Tizard, \& Whitmore, 1970), SNAP checklist, and the 
Conners Abbreviated Teacher Questionnaire; (c) short WISC-R using the Similarities, Vocabulary, Object Assembly, and Block Design subtests (Wechsler, 1974); and (d) Wide Range Achievement Test (WRAT; Jastak $\&$ Wilkinson, 1984) scores for reading and spelling. Subjects were considered normal only if ratings were within the range specified for normal subjects on all measures used. For the children attending the clinic, the diagnosis was based on information obtained from an interview of each child's parents, as well as the behavior ratings and psychoeducational assessment used for the normal controls.

Information was obtained by one of two child psychiatrists from each child's parent(s) by means of an interview designed to elicit symptoms relevant to DSM-III diagnosis. This interview was developed because the description of child behavior elicited through questionnaire responses or a structured interview was not detailed enough to distinguish symptoms of hyperactivity from those of conduct disorder (Schachar et al., 1986). The interview covered prenatal, birth, postnatal, developmental, medical, academic, and family histories as well as current child behavior and symptoms of psychopathology. It investigated (1) the quality of the child's interaction with peers, siblings, and adults, and (2) symptoms associated with affective, anxiety, and psychosomatic disorders. Special attention was paid to activity level, attentiveness, and impulsiveness in various settings. For each setting or symptom, parents were asked to describe a recent example of their child's behavior. Subjective comments were not considered; instead, we rated each symptom and, in arriving at a diagnosis, identified as symptoms only behaviors that were severe, handicapping, and inappropriate for the child's age.

Interrater reliability of this interview was assessed by having a second child psychiatrist complete ratings from an audiotape of 20 interviews. Raters agreed on the presence or absence of $97 \%$ of symptoms (Kappa $=.92$ ). In no case did disagreement about specific symptoms result in disagreement about diagnosis.

If the psychoeducational tests had been administered to the child within the previous year, the results were requested and the child was not retested.

Any child who had a full-scale intelligence quotient of less than 80 , evidence of a neurological disorder such as epilepsy, history of psychosis, or current treatment with medication was excluded from further study.

DSM-III Diagnosis. Attention deficit disorder with hyperactivity (ADDH) was diagnosed if parental interviews revealed that the child had at least three symptoms of impulsivity, three of inattentiveness, and two of overactivity from among those listed in DSM-III (American Psychiatric Association, 1980) and there was a history of overactivity, impulsivity, or inattentiveness antedating the age of 6 years. 
According to DSM-III, ADDH can be diagnosed on the basis of teacher report even when it is not in agreement with parental report. Accordingly, ADDH was diagnosed if a child's teacher rated him as disturbed and significantly hyperactive. A rating of 9 or more on the Rutter-B scale was taken as evidence of psychiatric disturbance (Rutter et al., 1970). Clinically significant hyperactivity was judged to be evident in the presence of any two of the following criteria: (a) a rating of at least 5 out of 6 on the Rutter-B hyperactivity factor, a score that is obtained by $3 \%$ of 10 -year-old boys (Schachar et al., 1981), (b) a rating of 4 inattentive, 4 impulsive, and 3 overactive items on the SNAP, a score that is obtained by $5 \%$ of 10 -year-old boys (W. E. Pelham, personal communication, 1981), (c) an abbreviated Conners score of 15 or more, a score that is predictive of a clinical diagnosis of hyperactivity (Goyette, Conners, \& Ulrich, 1978).

Children were assigned to the conduct disorder (CD) groups if they met DSM-III diagnostic criteria for conduct disorder or oppositional disorder. Oppositional disorder was diagnosed only in the presence of severe and pervasive oppositional symptoms involving relationships with both parents or a variety of adults. Conduct and oppositional disorders were combined on the grounds that the two diagnoses have not been differentiated and are qualitatively similar (Anderson, Williams, McGee, \& Silva, 1987; Werry et al., 1987; Werry, Methuen, Fitzpatrick, \& Dixon, 1983).

Learning disability (LD) was diagnosed if the child's WRAT reading and spelling scores were both at least 15 standard score points below his fullscale IQ in the absence of a physical, sensory, or emotional problem (Cohen \& Netley, 1978).

On the basis of these criteria, children were assigned to one of four diagnostic groups: ADDH, CD, mixed ADDH plus CD (ADDH $+\mathrm{CD})$, and learning disorder (LD). Children in the $\mathrm{CD}$ and $\mathrm{LD}$ groups were not hyperactive according to DSM-III criteria.

ICD-9 Diagnosis. In keeping with European clinical practice, the diagnosis of hyperkinetic syndrome (HS) was applied to the subset of patients with a diagnosis of ADDH who had evidence of clinically significant hyperactivity on both parental report and teacher ratings (Taylor, 1986). The ICD-9 criteria for clinically significant hyperactivity on each of these measures were similar to those used to establish DSM-III diagnosis. ICD-9 criteria for conduct and learning disorders were the same as for DSM-III diagnosis.

\section{Apparatus}

The stimuli for the CPT were presented visually using a Commodore 8032 or a PET 2001 computer. The stimuli consisted of 10 upper-case letters ( $A, C, D, G, L, M, N, O, T$, and $X$ ). The letter $X$ was designated as the tar- 
get. The letters presented using the Commodore 8032 were $2 \times 4 \mathrm{~mm}$ and those presented using the PET 2001 were $3 \times 4 \mathrm{~mm}$. Letters were presented one at a time in the center of the video screen. Each letter was programmed so that it occurred equally often (10\% of the time). As is usual in CPT research with children, a ratio of 10 trials per target was used. The order in which the letters were presented was randomly generated by the computer. A different random order was prepared for each subject. The three SOAs were 1, 2 , and $4 \mathrm{sec}$ and the three DTs $0.2,0.4$ and $0.8 \mathrm{sec}$. The number of letter presentations (trials) in each CPT condition was kept constant at 120 , so that the task length was manageable for the subjects.

\section{Procedure}

Written consent was obtained from the parents of all subjects and verbal assent from each child. Parents of patients were interviewed while the child was tested on the WISC-R, the WRAT, and the CPT. The entire procedure involved about 1 or 2 hours with the child and about the same amount of time with the parents.

Essentially the same procedure was followed with the controls, but there were some minor but necessary changes. For example, testing of children was carried out at the school instead of at the hospital. Parents of normal controls were not interviewed because permission to do so could not be obtained from the school or school board.

Subjects were seated 40 to $60 \mathrm{~mm}$ from the screen when testing began. Their viewing distance was not constrained, and they were free to move about as long as they held onto the board on which a telegraph key was mounted. Subjects were instructed to "catch as many of the $X$ s as you can, pushing the button as quickly as possible without making mistakes." They were told not to respond when other letters were presented.

For each subject the accuracy and RT of responses in each CPT condition were recorded by the computer. Subjects were given a short break of several minutes between CPT conditions, and the experimenter remained with each subject in the testing session at all times.

\section{Design}

The use of three levels of SOA and three levels of DT resulted in nine possible conditions on the CPT. Each subject was tested in all nine conditions. The order in which the subjects received each condition was counterbalanced using a Latin-square design. In addition, within each group, subjects were randomly assigned to the order in which they received each CPT con- 
dition. Performance was compared in terms of (a) probability of hits, (b) probability of false alarm responses (responding when a target was not present), and (c) mean RT for correct target detections (hits). Thus, it was a $5 \times 3$ $\times 3$ factorial design with repeated measures on SOA and DT. Because overall main effects and interactions might not be significant if only the hyperactive group differed, planned comparisons were conducted to assess differences between (a) hyperactive children (ADDH or HS group) and NC, (b) hyperactive patients (ADDH or HS group) and other pathological controls, and (c) ADDH or ADDH + CD groups.

We hypothesized that there would be a main effect of both SOA and DT. Such a result would confirm that the experimental procedure had manipulated attention. Also, interactions between diagnostic group and both SOA and DT were predicted. These two hypotheses address the possibility of an attention deficit associated with hyperactivity.

Planned comparisons were expected to show significant differences between hyperactive children and the NC group (i.e., a simple interaction). They were also expected to indicate significant differences between hyperactive children and pathological controls in the presence of an attention deficit that was specific to hyperactivity. If only the former expectation was met, one could conclude that attention deficit is a correlate of various forms of disturbance. A main effect for diagnostic groups was predicted but was not relevant to the issue of attention deficit.

\section{Statistics}

A 5 (diagnostic group) $\times 3(\mathrm{SOA}) \times 3(\mathrm{DT})$ mixed analysis of variance (ANOVA) with repeated measures for the latter two factors was carried out for each of the dependent variables of RT, hit rate, and false alarm rate. To adjust possible violations of compound symmetry, the Greenhouse Geiser correction was employed throughout (Kirk, 1982).

\section{Results}

There were no significant differences among diagnostic groups constituted according to DSM-III or ICD-9 criteria in either age or full-scale WISC-R scores (Table I).

\section{DSM-III Diagnosis}

Hit Rate. In general, subjects had fewer hits when stimuli were presented at 1 or $4 \mathrm{sec}$ intervals than at intervals of $2 \mathrm{sec}$. They also had significantly 
Table I. Characteristics of Each Diagnostic Group Defined Using DSM-III Criteria in Experiment $1^{a}$

\begin{tabular}{|c|c|c|c|c|c|c|c|c|c|c|}
\hline \multirow[b]{3}{*}{ Measure } & \multicolumn{10}{|c|}{ Diagnostic group (DSM-III criteria) } \\
\hline & \multicolumn{2}{|c|}{$\begin{array}{c}\text { ADDH } \\
(N=14)\end{array}$} & \multicolumn{2}{|c|}{$\begin{array}{c}\mathrm{CD} \\
(N=8)\end{array}$} & \multicolumn{2}{|c|}{$\begin{array}{l}\mathrm{ADDH}+\mathrm{CD} \\
(N=18) \\
\end{array}$} & \multicolumn{2}{|c|}{$\begin{array}{c}\text { LD } \\
(N=11)\end{array}$} & \multicolumn{2}{|c|}{$\begin{array}{c}\text { NC } \\
(N=36)\end{array}$} \\
\hline & Mean & $S D$ & Mean & $S D$ & Mean & $S D$ & Mean & $S D$ & Mean & $S D$ \\
\hline Age (months) & 95.3 & 16.5 & 3.4 & 20.3 & 101.8 & 16.5 & 108.5 & 17.0 & 102.9 & $19 . C$ \\
\hline Full-scale $\mathrm{IQ}^{\prime}$ & 105.3 & 10.7 & 108.1 & 12.4 & 108.4 & 13 & 105.4 & 14.3 & 109.6 & 12.7 \\
\hline RT & 806.7 & 84.7 & 706.6 & 142.7 & 728.0 & 186.4 & 693.2 & 141.1 & 691.0 & 164.3 \\
\hline Hit rate $(\%)$ & 71.2 & 28.0 & 87.1 & 18.8 & 87.1 & 17.8 & 92.5 & 11.9 & 91.1 & 13.2 \\
\hline \multicolumn{11}{|l|}{ False alarm } \\
\hline rat & 4.4 & 5.7 & 2.0 & 2.2 & 3.8 & 4.6 & 3.8 & 5.1 & 1.3 & 2.1 \\
\hline
\end{tabular}

${ }^{\alpha}$ Results for diagnoses using ICD-9 criteria are available on request.

fewer hits with a decreasing DT. This conclusion was supported by significant main effects of both SOA $(F(2,162)=9.54, p<0.01)$ and DT $(F(2$, $162)=14.26, p<0.01)$. Furthermore, with a short DT, the hit rate decreased significantly more as event rate slowed $(F(4,324)=5.10, p<0.01)$.

Hit rate deteriorated at fast and slow event rates in the ADDH group much more than in the other diagnostic groups. This was evident in the planned comparisons (ADDH vs. pathological controls, $F(2,162)=4.13$, $p<0.05$; ADDH vs. $\mathrm{NC}, F(2,164)=3.84, p<0.05)$ but the overall interaction between diagnostic group and SOA was nonsignificant $(F(8,164)$ $=1.32$, n.s.). Hit rates of the various DSM-III diagnostic groups were not affected differently by manipulation of DT.

The ADDH group performed less well than the other groups when the data for SOA and DT condition were collapsed. The ADDH group had the lowest and the NC and LD groups the highest mean hit rates $(F(4,81)=$ $6.52, p<0.01$ ) (Table I). Planned comparisons indicated that the hit rate of the ADDH group was significantly lower than the rates of the NC group $(F(4,81)=23.53, p<0.01)$ and the pathological controls $(F(4,81)=18.25$, $p<0.01$, see Figure 1a and Table I). For hit rate, the interaction involving SOA, DT, and diagnosis was not significant.

False Alarm Rate. As predicted, the false alarm rate was significantly higher when event rate was slow than when it was fast $(F(2,162)=21.72$, $p<0.01)$. It was also significantly higher when the DT was short than when it was long $(F(2,162)=6.68, p<0.01)$. Moreover, there was an interaction between SOA and DT: The false alarm rate increased more with slower event rates when the DT was short $(F(4,324)=3.54, p<0.01)$.

The ADDH group did not differ significantly from the $\mathrm{NC}$ group $(F(2$, $162)=1.99$, n.s.) or from the other pathological controls $(F(2,162)=0.62$, n.s.), although the false alarm rate was affected by variation in SOA in some groups significantly more than in others $(F(8,162)=2.18, p<0.05)$ (Figure 1b). The rate of false alarms among the groups did not differ significantly with variation in DT. 

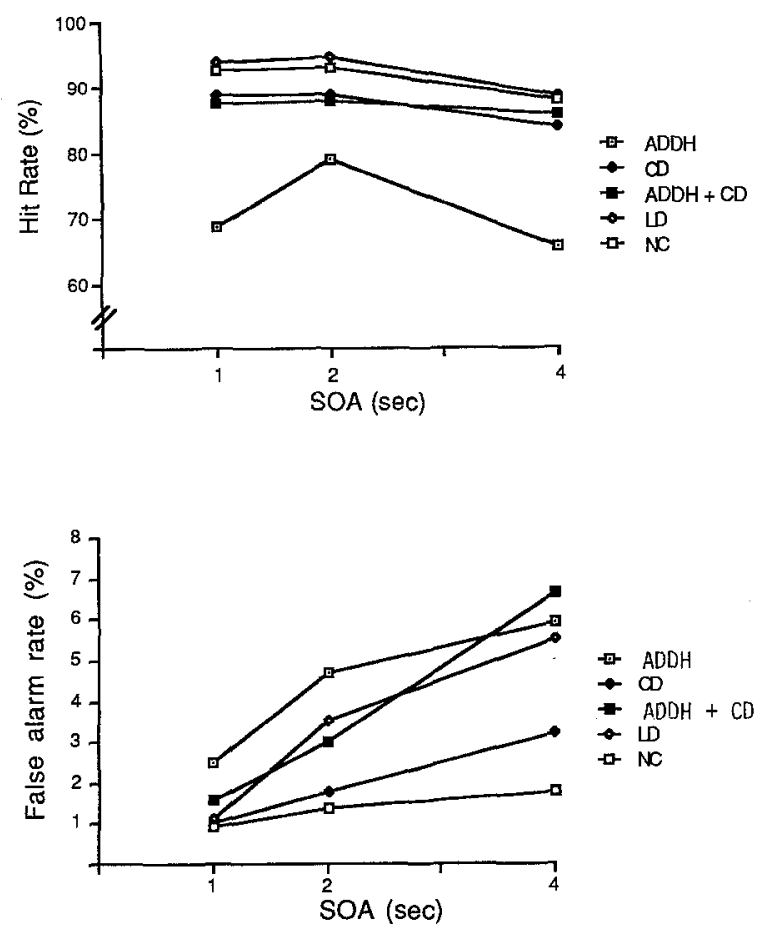

Fig. 1. DSM-III groups in Experiment 1. a. Mean hit rate (percentage) for each group on each SOA condition. $b$. Mean false alarm rate (percentage) for each group on each SOA condition.

A significant main effect for DSM-III diagnostic group was associated with false alarm rates $(F(4,81)=4.28, p<0.01)$. The ADDH group made significantly more false alarms than the NC group $(F(1,81)=11.97, p<$ $0.01)$, but not more than the pathological control groups $(F(1,81)=1.69$, n.s., Figure 1b). For false alarm rate, the interaction between SOA, DT, and DSM-III diagnostic group was not significant.

Reaction Time. RT increased as event rate slowed $(F(2,162)=37.53$, $p<0.01)$ and DT increased $(F(2,162)=15.24, p<0.01)$. There was a significant interaction between SOA and DT: RT increased with slower event rates more when DT was long $(F(4,324)=3.03, p<0.05)$.

For RT, neither the interaction between SOA, DT, and diagnosis, nor interactions between experimental group and SOA or DT were significant. Planned comparisons of ADDH and NC or ADDH and other pathological controls were also nonsignificant.

The main effect of DSM-III diagnostic group on mean RT was not significant $(F(4,81)=2.08$, n.s. $)$, but planned comparisons indicated that the 
RT of the ADDH group was significantly longer than that of the NC group $(F(1,81)=7.70, p<0.01)$ or of the pathological control groups $(F(1,81)$ $=5.07, p<0.05)$.

\section{ICD-9 Diagnosis}

The effect of variations in SOA and DT on hit rate, false alarm rate, and $\mathrm{RT}$, and the interaction of SOA and DT across ICD-9 diagnostic groups were similar to those seen with the DSM-III diagnostic groups.

Hit Rate. The hit rate of the ICD-9 diagnostic groups was not affected differently by manipulation of SOA or DT. Nor was the overall interaction of SOA, DT, and diagnosis significant. However, the ICD-9 diagnostic groups differed across all SOA and DT conditions in the hit rate $(F(4,76)=4.38$, $p<0.01$ ), with the HS group detecting significantly fewer targets $(66.4 \%)$ than the NC group $(91 \%,(F(1,76)=17.49, p<0.01)$ or the pathological control groups $(84 \%,(F(1,76)=12.63, p<0.01)$.

False Alarm Rate. The false alarm rate was affected by variation in SOA more in the HS group than in the other diagnostic groups (Figure 2a). The overall interaction for false alarm rate between SOA and ICD-9 diagnostic groups was not significant $(F(8,152)=1.98$, n.s.). However, planned comparisons indicated that, with slower event rates, performance in the HS group deteriorated significantly more than in the NC group $(F(2,152)=3.85$, $p<0.05$ ) but not significantly more than in the pathological comparison groups $(F(2,152)=0.89$, n.s. $)$.

False alarm rate was affected significantly more by variation of DT in the HS group than in the other groups $(F(8,152)=2.21, p<0.05)$ (Figure $2 b)$. The decline in performance at the shortest DT was particularly marked for the HS group, which was significantly more affected by variation in DT than was the NC group $(F(2,152)=7.40, p<0.01)$ or the pathological control groups $(F(2,152)=4.36, p<0.05)$. For false alarm rate, the interaction of SOA, DT, and ICD-9 diagnosis was not significant. The mean false alarm rate of the ICD-9 diagnostic groups across all SOA and DT conditions also differed significantly $(F(4,76)=6.55, p<0.01)$, with the HS group making more false alarms than the NC group $(F(1,76)=23.0, p<0.01)$ or pathological controls $(F(1,76)=11.08, p<0.01)$, see Figure $2 \mathrm{a})$.

Reaction Time. RT was affected by variation in DT more in the HS group than in the other groups. There was no significant overall effect on RT between ICD-9 diagnostic group and DT. However, planned comparisons indicated that RT increased with increasing DT significantly more in the HS group than in the NC group $(F(2,152)=4.05, p<0.05)$ or the pathological controls $(F(2,152)=4.30, p<0.05)$ (Figure $2 c)$. The interaction of SOA, DT, and diagnostic group for RT was not significant. The mean 

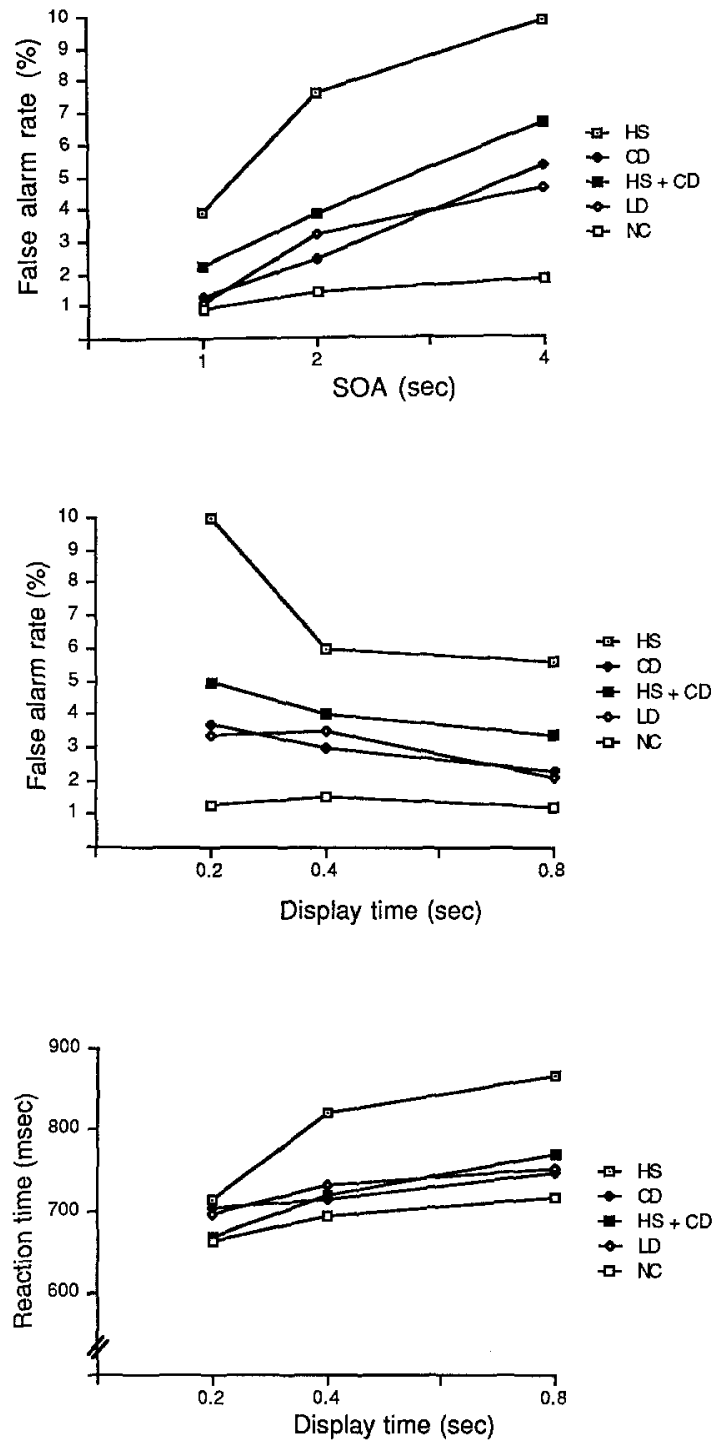

Fig. 2. ICD-9 groups in Experiment 1. a. Mean false alarm rate (percentage) for each group on each SOA condition. b. Mean false alarm rate (percentage) for each group for each DT. c. Mean RT for hits by each group for each DT. 
RT of the ICD-9 diagnostic group across all SOA and DT conditions did not differ significantly.

\section{DISCUSSION}

The effects of event rate and DT on RT and accuracy (as identified by hit and false alarm rates) were consistent with expectations derived from attention theory and from most research with vigilance tasks. A faster RT and inferior accuracy rate were associated with a shorter DT, a slower event rate with a longer RT and a higher false alarm rate, and a lower hit rate with both the slowest and the fastest SOA.

In addition, and central to the issue of attention deficit, performance of hyperactive subjects (ADDH or HS) was uniquely affected by the variation in demand for attention imposed by different SOAs and DTs. Compared with the 2-sec SOA, both rapid (1-sec) and slow (4-sec) stimulus presentations were more detrimental to the performance of children in the ADDH group than in the other groups. Variation in SOA also affected performance in the HS group more than in the NC group but not more than in the pathological control groups. More attention-demanding SOA conditions were associated with a decreasing hit rate and unchanged false alarm rates in the ADDH group, and an increasing false alarm rate and unchanged hit rate in the HS group. These changes in performance reflect altered sensitivity, not strategic fluctuation or criterion adjustment.

Manipulation in DT distinguished between ICD-9 diagnotic groups but not between DSM-III diagnostic groups. For the HS group the most attentiondemanding DT condition was associated with an increasing false alarm rate without a corresponding change in hit rate, indicative of an underlying lack of sensitivity.

These results replicated those of earlier CPT and RT research, which showed that patients with ADDH generally perform less well than NC. On our CPT task, the ADDH group detected fewer targets, made more false alarms, and had slower RT than the NC or pathological control groups. These findings suggested that some general performance shortcoming may be present in ADDH, in addition to a deficit in sustained attention.

Our results identified several differences in the predictive validity of the two diagnostic schemata used in the study. The performance of the 6 children in the HS group was unique in more ways than that of the 14 children assigned a DSM-III diagnosis of ADDH. The performance of both groups was more adversely affected by the attention demand imposed by variation in SOA, but only the HS group was uniquely affected by the attention demand imposed by the shortest DT.

The performance of the HS group was also inferior to that of the ADDH group over all conditions. The 6 HS patients detected a lower per- 
centage of targets than the 14 ADDH subjects $(66 \%$ vs. $71 \%)$ and made more false alarms ( $7 \%$ vs. $4 \%$ ). Whereas children in the ADDH group made more false alarms than the NC but not more than the pathological controls, the HS patients had more false alarms than any control groups.

In this experiment, SOA was confounded with time on task: Because the number of trials was kept constant across SOA conditions, slower event rates were associated with longer task conditions. Deterioration in performance with length of time on task is characteristic of hyperactive children (Cohen \& Douglas, 1972) and of vigilance tasks in general (Parasuraman, 1984) and the CPT in particular. Therefore, it was necessary to conduct another experiment in which length of time on task was held constant to determine whether the SOA effects observed in this experiment were due to task duration.

\section{EXPERIMENT 2}

The purpose of Experiment 2 was to distinguish the effects of SOA and time on task in Experiment 1.

\section{Method}

\section{Subjects}

The subjects were 21 boys with learning or psychiatric disorders and 36 normal boys. Their ages ranged from 6 to 12 years.

The clinic patients included 19 boys who had been subjects in Experiment 1 . The two additional subjects were selected and diagnosed using the same criteria and procedures. The clinically disturbed children involved in this second experiment belonged to the same diagnostic categories as those in Experiment 1. All 36 normal children participated in both experiments. When subjects served in both experiments, Experiment 1 always preceded Experiment 2, and the sessions were at least 2 weeks apart to minimize the possibility of specific practice effects. There were no significant differences in full-scale WISC-R scores or mean age between the DSM-III diagnostic groups (Table II). In this experiment there were insufficient children with ICD-9 diagnosis of HS to permit analysis.

\section{Apparatus and Procedure}

The apparatus for Experiment 2 was similar to that of Experiment 1 except that a different set of upper-case letters $(B, F, I, K, P, Q, R, V, W$, 
Table II. Characteristics of Each Diagnostic Group Defined Using DSM-III Criteria in Experiment 2

\begin{tabular}{|c|c|c|c|c|c|c|c|c|c|c|}
\hline \multirow[b]{3}{*}{ Measure } & \multicolumn{10}{|c|}{ Diagnostic group (DSM-III criteria) } \\
\hline & \multicolumn{2}{|c|}{$\begin{array}{c}\text { ADDH } \\
(N=4)\end{array}$} & \multicolumn{2}{|c|}{$\begin{array}{c}\mathrm{CD} \\
(N=5)\end{array}$} & \multicolumn{2}{|c|}{$\begin{array}{l}\mathrm{ADDH}+\mathrm{CD} \\
(N=5) \\
\end{array}$} & \multicolumn{2}{|c|}{$\begin{array}{c}\text { LD } \\
(N=7)\end{array}$} & \multicolumn{2}{|c|}{$\begin{array}{c}\mathrm{NC} \\
(N=36)\end{array}$} \\
\hline & Mean & $S D$ & Mean & $S D$ & Mean & $S D$ & Mean & $S D$ & Mean & $S D$ \\
\hline Age (months) & 96.0 & 14.3 & 93.0 & 17.0 & 96.4 & 12.7 & 111.6 & 14.3 & 103.4 & 19.8 \\
\hline Full-scale IQ & 108.0 & 11.9 & 110.8 & 7.8 & 110.8 & 9.6 & 98.0 & 5.9 & 109.6 & 12.7 \\
\hline RT & 919.3 & 129.9 & 770.1 & 184.9 & 706.3 & 131.9 & 774.4 & 131.1 & 789.9 & 150.6 \\
\hline Hit rate $(0 \%)$ & 54.8 & 18.3 & 77.7 & 22.2 & 71.8 & 30.3 & 80.2 & 20.0 & 84.2 & 18.3 \\
\hline $\begin{array}{l}\text { False alarm } \\
\text { rate }(\%)\end{array}$ & 4.8 & 3. & 5.0 & 3.1 & 4.3 & 3.4 & 3.6 & 5.2 & 1.2 & 1.4 \\
\hline
\end{tabular}

and $Z$ ) and a different target letter $(Z)$ were used. The letter and the target were different from those in Experiment 1 to minimize specific practice effects for subjects who took part in both experiments. Unlike Experiment 1, the number of stimuli presented varied with each condition. This was inevitable since task duration was fixed at 16 min while event rate varied. The number of trials was 960 for SOA $1 \mathrm{sec}, 480$ for SOA $2 \mathrm{sec}$, and 240 for SOA $4 \mathrm{sec}$. Consequently, many more trials were presented in Experiment 2 than in Experiment 1.

The procedure, design, and analysis were identical to those of Experiment 1 except that the use of three levels of SOA and one level of DT (0.4 sec) resulted in three possible conditions on the CPT.

\section{Hypothesis}

We hypothesized that the effects of SOA noted in Experiment 1 would be replicated in Experiment 2 only if they were due to SOA rather than time on task.

\section{Results}

\section{DSM-III Diagnosis}

Hit Rate. Hit rate was not significantly affected by variation in SOA. Nor were the diagnostic groups differently affected by manipulation of SOA. The poorer performance of the ADDH group was confirmed by these data. The ADDH group detected fewer targets than the NC group (55\% vs. 84\%), $(F(1,52)=9.44, p<0.01)$ or the pathological control groups $(F(1,52)=$ $4.83, p<0.05)$.

False Alarm Rate. The false alarm rate was significantly affected by variation in $\operatorname{SOA}(F(2,104)=12.16, p<0.01)$, with a slower event rate 


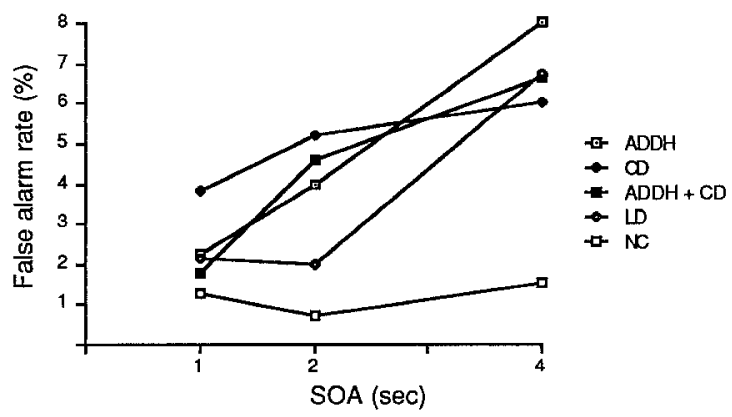

Fig. 3. DSM-III groups in Experiment 2: Mean false alarm rate (percentage) for each group on each SOA condition.

being associated with a greater false alarm rate. As in Experiment 1, a significant interaction between SOA and diagnostic groups was found $(F(2,104)$ $=2.20, p<0.05$, Figure 3 ). As the event rate slowed, the false alarm rate increased more in the ADDH group than the $\operatorname{NC}$ group $(F(2,104)=5.32$, $p<0.05)$ but not more than in the pathological comparison groups $(F(2$, $104)=0.32$, n.s.). Over all SOA conditions, the ADDH group made significantly more false alarms than the $\mathrm{NC}$ group $(F(1,52)=9.36, p<0.01)$.

Reaction Time. RT was significantly affected by $\operatorname{SOA}(F(2,104)=$ $20.21, p<0.01$ ), with a slower event rate being associated with a slower RT. For RT, the interaction between SOA and diagnostic group was not significant. The ADDH group had a slower RT than the pathological control groups $(F(1,52)=5.01, p<0.05)$ but not slower than the NC group $(F(1$, $52)=3.37$, n.s.).

\section{Discussion}

Experiment 2 confirmed that SOA affected attention to the task as measured by the RT for hits and the false alarm rate. The findings also replicated those of Experiment 1, indicating that the SOA effects observed in Experiment 1 for RT and false alarm rate were probably not due to task duration. Instead, the SOA effect continued to be significant even when task duration was controlled for and extended from that used in Experiment 1. In contrast, we found that event rate had no effect on hit rate, indicating that the change in hit rate observed in Experiment 1 can probably be attributed to task duration.

The increase in false alarms and RT with a slower event rate (increasing SOA) indicated that subjects do not maintain their attention on the task as much as when stimuli are presented in rapid succession. The fact that this 
increase in false alarms occurs without a corresponding decrease in RT contradicts the proposition that subjects adjust the trade-off between speed and accuracy with changes in SOA. And the absence of a corresponding increase in hits indicates that the decrement with increasing SOA is not a result of a shift in response criterion.

The second experiment revealed that the effects of the event rate on the hit rate of the various diagnostic groups did not differ when length of time on task was controlled. The increase in the false alarm rate with a slower event rate seen in the ADDH group compared with the NC group, found in Experiment 1, was replicated in Experiment 2, where task duration was controlled. However, this effect was not unique to the ADDH group. Because DT was not confounded with task length in Experiment 1, the unique effects of variation in DT on the performance of the HS group cannot be explained as a function of task duration.

Also, this second experiment replicated the finding that the performance of patients with $\mathrm{ADDH}$ was poorer than that of the NC group. As in Experiment 1 , the patients with ADDH had significantly less good hit and false alarm rates than the NC group and significantly slower RT and fewer hits than the pathological control groups.

\section{GENERAL DISCUSSION}

Demand for sustained attention was manipulated successfully in these experiments by varying SOA and DT. As predicted, slower event rates (longer SOA) were associated with a slower RT, a greater number of false alarms, and fewer correct detections. A shorter DT was associated with a faster RT, a lower hit rate, and more false alarms. The combination of a lower hit rate and a higher false alarm rate suggested a general decline in sensitivity of the subject with more attention-demanding conditions (i.e., fastest and slowest event rates and shortest DT).

In Experiment 1, the ADDH group, like most groups, performed better at an SOA of $2 \mathrm{sec}$ than at $4 \mathrm{sec}$. The poor performance at the SOA of $4 \mathrm{sec}$ may reflect a waning of attention or a tendency for attention to wander between stimuli. This effect may be stronger with ADDH patients than with clinical and normal controls, but it was observed to a greater or lesser extent in all groups of subjects. Because this decreasing hit rate at a longer SOA was associated with an increasing false alarm rate, it could also be attributed to a decline in sensitivity.

The poor performance at an SOA of $1 \mathrm{sec}$ is more interesting because it is specific to ADDH patients. It may reflect a refractory effect from processing the immediately preceding stimulus (Kantowitz, 1974; Welford, 1952); 
subjects may have been so occupied with the preceding stimulus that they missed the current one. The interaction suggests that subjects with ADDH may be more susceptible to refractory effects, or have refractory effects that extend over longer periods, than normal controls or disturbed children who do not have ADDH.

In Experiment 2, where time-on-task was the same for each SOA condition, there was no evidence that performance was poorer at an SOA of $1 \mathrm{sec}$. However, there were substantially more trials in the 1-sec SOA condition than in Experiment 1 (960 vs. 120), so the refractory effect may have been eliminated by practice (Logan, 1979). It would be worthwhile examining refractory effects in patients with ADDH more directly. The contrast between the two experiments suggests that practice may be an important variable.

As expected, the ICD-9 diagnosis of HS applied to less than half of the children diagnosed as having ADDH according to DSM-III. Therefore, the sample size was very small in the second experiment and conclusions about the relative predictive validity of these two approaches to diagnosis must be tentative.

Patients with HS responded to changes in DT differently than did children in the other groups. Their RT increased more as DT increased, from a level roughly equivalent to the other groups at a DT of $0.2 \mathrm{sec}$ to a level more than $100 \mathrm{msec}$ longer than the other groups at a DT of $0.8 \mathrm{sec}$. At the same time, HS patients made more than twice as many false alarms as the other children at $0.2 \mathrm{sec}$ DT, and decreased to levels much closer to the other children as DT increased.

This pattern suggests that HS patients may have traded speed for accuracy in the 0.2 -sec DT condition. Like other children, they responded more rapidly, as if they were paying closer attention. But unlike other children, their false alarm rates increased substantially, which suggests a shift in speed-accuracy criteria rather than more careful attention. This speed-accuracy trade-off may reflect the impulsiveness of children who have HS.

In our study, both the HS and the ADDH diagnoses delineated groups of children with poorer CPT performance than controls. However, the performance of the HS group was even worse than that of the ADDH group. Moreover, the CPT performances of the two groups were affected differently by demand for sustained attention imposed by variation in SOA and DT. The performance of the ADDH group was adversely affected by a slow event rate but not by a short DT, whereas that of the HS group was adversely affected by both conditions. This suggests a more global deficit in sustained attention among patients with HS. These findings also demonstrate another difference in the predictive validity of pervasive and situational hyperactivity: Pervasive hyperactivity is associated with more marked academic underachievement, poorer prognosis, and an earlier onset of symptomatology than 
situational hyperactivity (Sandberg, Rutter, \& Taylor, 1978; Schachar et al., 1981; Taylor, 1986; Thorley, 1984).

In addition, our results confirm previous studies showing that hyperactive children perform less well on the CPT than do normal controls (Klorman et al., 1983; Michael, Klorman, Salzman, Borgstedt, \& Dainer, 1981; Nuechterlein, 1983; Sykes et al., 1971). In particular, our results replicated those of Sykes et al. (1971), the only other study to have manipulated SOA. Both studies showed that the disadvantage of hyperactive children relative to normal controls increases with SOA length. However, we did not show the same effect of SOA overall. Sykes and colleagues (1971) found that perfomance improved with a longer SOA, while we found that it deteriorated. This discrepancy could be a result of differences in the DT between these two studies. In our study, the effect of SOA was greater with a shorter DT and less pronounced with a longer DT. Sykes and associates (1971) do not specify DT; possibly they employed a longer DT, in which case, the effects of longer SOA would be diminished.

Our findings in these experiments point to an unexpected difference between the ADDH and the ADDH + CD groups, with the former showing a more marked cognitive deficit. However, we have observed similar differences in other studies. In another sample, we found that these two diagnostic groups also have different inhibitory control. Children with a diagnosis of ADDH found it significantly more difficult than those with a diagnosis of mixed ADDH $+\mathrm{CD}$ to inhibit a motor response when instructed to do so (unpublished data). In yet another sample, we found that children with a diagnosis of ADDH $+\mathrm{CD}$ but not those with a diagnosis of ADDH had parents with higher rates of psychopathology than the parents of normal controls. The families of children in this mixed $\mathrm{ADDH}+\mathrm{CD}$ group were also more dysfunctional and more characterized by adversity than the families of ADDH subjects (unpublished data). The distinction is clearly important and requires further study.

In summary, the poor performance of hyperactive children on the CPT, which might be attributable to a unique deficit in sustained attention, was replicated in this study. The deficit might be associated with a greater susceptibility of hyperactive children to refractory effects or to differences in the way that they trade speed for accuracy. Differences between situationally and pervasively hyperactive children were noted and indicated that the HS group might have a more global deficit in sustained attention.

\section{REFERENCES}


Anderson, J. C., Williams, S., McGee, R., \& Silva, P. A. (1987). DSM-III disorders in preadolescent children: Prevalence in a large sample from the general population. Archives of General Psychiatry, 44, 69-76.

Campbell, S. B., \& Werry, J. S. (1986). Attention deficit disorder (hyperactivity). In H. C. Quay \& J. S. Werry (Eds.), Psychopathological disorders of childhood. New York: Wiley.

Cohen, N. J., \& Douglas, V. I. (1972). Characteristics of the orienting response in hyperactive and normal children. Psychophysiology, 9, 238-245.

Cohen, R. L., \& Netley, C. (1978). Cognitive deficits, learning disabilities, and WISC verbalperformance consistency. Developmental Psychology, 14, 624-634.

Conners, C. K. (1973). Rating scales in drug studies with children. Pharmacotherapy of Children (special issue), 24-29.

Davis, R. (1957). The human operator as a single channel information system. Quarterly Journal of Experimental Psychology, 9, 119-129.

Douglas, V. I. (1983). Attentional and cognitive problems. In M. Rutter (Ed.), Developmental neuropsychiatry (pp. 280-329). New York: Guilford Press.

Firestone, P., \& Martin, J. E. (1979). An analysis of the hyperactive syndrome: A comparison of hyperactive, behavior problem, asthmatic, and normal children. Journal of Abnormal Child Psychology, 7, 261-273.

Goyette, C. H., Conners, C. K., \& Ulrich, R. F. (1978). Normative data on revised Conners Parent and Teacher Rating Scales. Journal of Abnormal Child Psychology, 6, 221-236.

Jastak, S., \& Wilkinson, G. S. (1984). The Wide Range Achievement Test Revised Administration Manual. Wilmington, DE: Jastak.

Kantowitz, B. H. (1974). Double stimulation. In B. H. Kantowitz (Ed.), Human information processing: Tutorials in performance and cognition (pp. 83-131). Hillsdale, NJ: Erlbaum.

Kirk, R. E. (1982). Experimental design. California: Brooks-Cole.

Klorman, R., Salzman, L. F., Bauer, L. O., Coons, H. W., Borgstedt, A. D., \& Halpern, W. I. (1983). Effects of two doses of methylphenidate on cross-situational and borderline hyperactive children's evoked potentials. Electroencephalography and Clinical Neurophysiology, 56, 169-185.

Lambert, N. M., \& Sandoval, J. (1980). The prevalence of learning disabilities in a sample of children considered hyperactive. Journal of Abnormal Child Psychology, 8, 33-50.

Logan, G. D. (1979) On the use of a concurrent memory load to measure attention and automaticity. Journal of Experimental Psychology, 5, 189-207.

McGrath, J. J., Harabedian, A., \& Bruckner, D. N. (1968). Review and critique of the literature on vigilance performance. In Studies of human vigilance: An omnibus of technical reports. Goleta, CA: Human Factors Research.

Michael, R. L., Klorman, R., Salzman, L. F., Borgstedt, A. D., \& Dainer, K. B. (1981). Normalizing effects of methylphenidate on hyperactive children's vigilance performance and evoked potentials. Psychophysiology, 18, 665-677.

Nuechterlein, K. H. (1983). Signal detection in vigilance tasks and behavioral attributes among offspring of schizophrenic mothers and among hyperactive children. Journal of Abnormal Psychology, 92, 4-28.

Parasuraman, R. (1984). Sustained attention in detection and discrimination. In R. Parasuraman \& D. R. Davies (Eds.), Varieties of attention (pp. 243-271). Orlando: Academic Press.

Prior, M., \& Sanson, A. (1986). Attention deficit disorder with hyperactivity: A critique. Journal of Child Psychology and Psychiatry, 27, 307-319.

Rosvold, H. E., Mirsky, A. F., Sarason, I., Bransome, E. D., Jr., \& Beck, L. H. (1956). A continuous performance test of brain damage. Journal of Consulting Psychology, 20, 343-350.

Rutter, M. (1967). A children's behavior questionnaire for completion by teachers: Preliminary findings. Journal of Child Psychology and Psychiatry, 8, 1-11.

Rutter, M., Tizard, J., \& Whitmore, K. (1970). Education, health and behavior: Psychological and medical study of childhood development. New York: Wiley.

Sandberg, S. T., Rutter, M., \& Taylor, E. (1978). Hyperkinetic disorder in psychiatric clinic attenders. Developmental Medicine and Child Neurology, 20, 279-299.

Schachar, R., Rutter, M., \& Smith, A. (1981). The characteristics of situationally and pervasively hyperactive children: Implications for syndrome definition. Journal of Child Psychology and Psychiatry, 22, 375-392. 
Schachar, R., Sandberg, S., \& Rutter, M. (1986). Agreement between teachers' ratings and observations of hyperactivity, inattentiveness, and defiance. Journal of Abnormal Child Psychology, 14, 331-345.

Sergeant, J. A. (1981). Attentional studies in hyperactivity. Doctoral dissertation, University of Groningen, The Netherlands.

Sergeant, J. A., \& Scholten, C. A. (1985). On resource strategy limitations in hyperactivity: Cognitive impulsivity reconsidered. Journal of Child Psychology and Psychiatry, 26, 97-109.

Shaffer, D., McNamara, N., \& Pincus, J. H. (1974). Controlled observations on patterns of activity, attention, and impulsivity in brain-damaged and psychiatrically disturbed boys. Psychological Medicine, 4, 4-18.

Shaffer, L., \& Greenhill, D. (1979). A critical note on the predictive validity of "The Hyperkinetic Syndrome." Journal of Child Psychology and Psychiatry, 20, 61-72.

Swanson, , H. L. (1983). A developmental study of vigilance in learning-disabled and nondisabled children. Journal of Abnormal Child Psychology, 11, 415-429.

Sykes, D. H., Douglas, V.I., Weiss, G., \& Minde, K. K. (1971). Attention in hyperactive children and the effect of methylpehnidate (Ritalin). Journal of Child Psychology and Psychiatry, 12, 129-139.

Taylor, E. A. (1986). Overactivity, hyperactivity and hyperkinesis: Problems and prevalence. In E. A. Taylor (Ed.), The overactive child (pp. 1-18). Oxford: Blackwell Scientific.

Thorley, G. (1984). Review of follow-up and follow-back studies of childhood hyperactivity. Psychological Bulletin, 96, 116-132.

Wechsler, D. (1974). Wechsler Intelligence Scale for Children: Manual (revised). New York: Psychological Corporation:

Welford, A. T. (1952). The "psychological refractory period" and the timing of high-speed performance-A review and a theory. British Journal of Psychology, 43, 2-19.

Werry, J. S., Methven, R. J., Fitzpatrick, J., \& Dixon, H. (1983). The interrater reliability of DSM-III in children. Journal of Abnormal Child Psychology, 11, 341-354.

Werry, J. S., Reeves, J. C., \& Elkind, G. S. (1987). Attention deficit, conduct, oppositional, and anxiety disorders in children: I. A review of research on differentiating characteristics. Journal of the American Academy of Child and Adolescent Psychiatry, 26, 133-143.

World Health Organization (1978). Mental disorders: Glossary and guide to their classification in accordance with the ninth revision of the International Classification of Diseases. Geneva: Author. 